\title{
miR-210 over-expression enhances mesenchymal stem cell survival in an oxidative stress environment through antioxidation and c-Met pathway activation
}

\author{
XU JianFeng ${ }^{1,2,3 \dagger}$, HUANG ZheYong ${ }^{1 \dagger}$, LIN Li $^{1}$, FU MingQiang ${ }^{1}$, GAO YanHua ${ }^{1}$, \\ SHEN YunLi ${ }^{1}$, ZOU YunZeng ${ }^{1,2}$, SUN AiJun ${ }^{1,2}$, QIAN JuYing ${ }^{1 *} \&$ GE JunBo ${ }^{1,2^{*}}$ \\ ${ }^{1}$ Department of Cardiology, Zhongshan Hospital, Fudan University, Shanghai 200032, China; \\ ${ }^{2}$ Institutes of Biomedical Sciences, Fudan University, Shanghai 200032, China; \\ ${ }^{3}$ Department of Cardiology, Central Hospital of Minhang District, Shanghai 201199, China
}

Received March 26, 2014; accepted April 19, 2014; published online August 27, 2014

\begin{abstract}
microRNA-210 (miR-210) has generally been reported to be associated with cell survival under hypoxia. However, there are few data regarding the role of miR-210 in the survival of mesenchymal stem cells (MSCs) under oxidative stress conditions. Thus, we sought to investigate whether miR-210 over-expression could protect MSCs against oxidative stress injury and what the primary mechanisms involved are. The results showed that over-expression of miR-210 significantly reduced the apoptosis of MSCs under oxidative stress, accompanied by obvious increases in cell viability and superoxide dismutase activity and remarkable decreases in malonaldehyde content and reactive oxygen species production, resulting in a noticeable reduction of apoptotic indices when compared with the control. Moreover, the above beneficial effects of miR-210 could be significantly reduced by c-Met pathway repression. Collectively, these results showed that miR-210 over-expression improved MSC survival under oxidative stress through antioxidation and c-Met pathway activation, indicating the potential development of a novel approach to enhance the efficacy of MSC-based therapy for injured myocardium.
\end{abstract}

microRNAs, stem cells, oxidative stress, signal transduction

Citation: $\quad$ Xu JF, Huang ZY, Lin L, Fu MQ, Gao YH, Shen YL, Zou YZ, Sun AJ, Qian JY, Ge JB. miR-210 over-expression enhances mesenchymal stem cell survival in an oxidative stress environment through antioxidation and c-Met pathway activation. Sci China Life Sci, 2014, 57: 989-997, doi: $10.1007 / \mathrm{s} 11427-014-4725-\mathrm{z}$

Mesenchymal stem cells (MSCs) derived from bone marrow have become therapeutically important agents for injured myocardium repair because of their ability to differentiate into various cell types and to secrete protective factors [1]. However, the poor survival of transplanted MSCs greatly hampers their therapeutic efficacy $[2,3]$; this is mainly because the microenvironments that transplanted MSCs are exposed to often have various features that pro-

$\dagger$ Contributed equally to this work

*Corresponding author (email: qian.juying@zs-hospital.sh.cn; ge.junbo@zs-hospital.sh.cn) mote cell apoptosis, including oxidative stress (such as superoxide anions and hydrogen peroxide), hypoxia and inflammatory reactions [4]. An increasing number of studies have mentioned that excessive accumulation of reactive oxygen species (ROS) constantly pose a direct threat to cells via damage of the cell membrane, protein and DNA, leading to an alteration or loss of cellular functions $[5,6]$. Therefore, a strategy that protects MSCs against oxidative stress-induced injury might help to provide a cell-based treatment for heart disease.

microRNAs (miRNAs, miRs), through a combination of 
translational blockade and/or mRNA degradation, have been widely reported to possess the potential to regulate pathological and physiological processes [7], including proliferation, differentiation, death, metabolism and oxidative stress. It is currently believed that thousands of classic (protein-encoding) genes are controlled via such mechanisms; furthermore, it has been shown that some miRNAs tend to be associated with some special functional regulatory mechanisms [8], such as miR-146a for inflammatory response [9] and miR-126 for angiogenesis modulation [10]. In particular, miR-210 has been previously reported to be involved in oxidative stress and apoptotic defense [11]; for example, the blockade of miR-210 in myoblasts greatly increased myotube sensitivity to oxidative stress and mitochondrial dysfunction [12]. However, whether miR-210 plays a role in the apoptosis of MSCs exposed to oxidative stress and, if so, the underlying mechanism requires investigation.

Therefore, we compared the viability of MSCs overexpressing miR-210 with the control, and we examined the signal pathways that were potentially involved. Because the c-Met pathway is a key mechanism associated with cell apoptosis [13], we focused on the change of c-Met phosphorylation during this process. Additionally, we examined the activity of superoxide dismutase (SOD) [14], the level of malonaldehyde (MDA) [15], and the accumulation of ROS [16], which are reliable indicators for cellular oxidative stress. Hydrogen peroxide $\left(\mathrm{H}_{2} \mathrm{O}_{2}\right)$, widely used as an oxidant in vitro, was applied to develop a cellular model of oxidative stress damage.

\section{Materials and methods}

All animal treatments were performed in accordance with the Guidelines for the Care and Use of Laboratory Animals, published by the National Academy Press (NIH Publication No. 85-23, Revised 1996). The studies were approved by the Animal Care and Use Committee of Zhongshan Hospital, Fudan University.

\subsection{Isolation and culture of MSCs}

MSCs were derived from 4-week-old Sprague-Dawley (SD) rats according to the methods described in our previous study [17]. Briefly, the bone marrow in femurs and tibias was flushed with Dulbecco's modified Eagle's medium (DMEM; GIBCO, USA), 10\% fetal bovine serum (FBS; GIBCO) and penicillin/streptomycin. After incubation at $37^{\circ} \mathrm{C}$ in an atmosphere of $5 \% \mathrm{CO}_{2}$, the culture medium of bone marrow solution was changed every 3-4 d, and the spindleshaped, adherent MSCs were isolated and expanded. Passage 4 MSCs were used for subsequent experiments.

\subsection{In vitro exposure to $\mathrm{H}_{2} \mathrm{O}_{2}$}

MSCs were cultured with DMEM supplemented with $1 \%$ FBS for $6 \mathrm{~h}$ prior to experimental interventions. The starved MSCs were then exposed to $\mathrm{H}_{2} \mathrm{O}_{2}\left(0-1000 \mu \mathrm{mol} \mathrm{L}^{-1}\right)$ for $24 \mathrm{~h}$. The subsequent experiments were performed in four groups: the MSCs group (the control group); the MSCs ${ }^{\text {miR-210 }}$ group (MSCs over-expressing miR-210); the $\mathrm{H}_{2} \mathrm{O}_{2}$ group (MSCs incubated with $400 \mu \mathrm{mol} \mathrm{L} \mathrm{L}^{-1} \mathrm{H}_{2} \mathrm{O}_{2}$ for $6 \mathrm{~h}$ ); and the $\mathrm{MSCs}^{\mathrm{miR}-210}+\mathrm{H}_{2} \mathrm{O}_{2}$ group (MSCs ${ }^{\text {miR-210 }}$ exposed to $400 \mu \mathrm{mol}$ $\mathrm{L}^{-1} \mathrm{H}_{2} \mathrm{O}_{2}$ for $6 \mathrm{~h}$ ). To explore the mechanisms involved, three additional groups were added as follows: the MSCs ${ }^{\mathrm{miR}-210}+\mathrm{H}_{2} \mathrm{O}_{2}+\mathrm{SU} 11274$ and $\mathrm{MSCs}^{\mathrm{miR}-210}+\mathrm{H}_{2} \mathrm{O}_{2}+$ DMSO groups (MSCs ${ }^{\text {miR-210 }}$ was pre-incubated with a c-Met inhibitor (SU11274, $10 \mu \mathrm{mol} \mathrm{L}{ }^{-1}$ ) or vehicle (DMSO), respectively, for $1 \mathrm{~h}$ prior to treatment with $\mathrm{H}_{2} \mathrm{O}_{2}$ as above) and the MSCs+SU11274 group (MSCs were only incubated with $10 \mu \mathrm{mol} \mathrm{L}{ }^{-1}$ SU11274). The concentration of SU11274 (CalBiochem, USA) adopted in these experiments was determined from pilot studies (data not shown) and based on similar methods used in the literature [18].

\subsection{MSC viability assay}

The 3-(4,5-dimethylthiazol-2-yl)-2,5-diphenyltetrazolium bromide (MTT; Sigma, USA) assay was used to detect the viability of MSCs, which were treated with different concentrations of $\mathrm{H}_{2} \mathrm{O}_{2}$ in prescribed concentrations as follows: $0,100,200,400$ and $1000 \mu \mathrm{mol} \mathrm{L}{ }^{-1}$ at $37^{\circ} \mathrm{C}$ and $5 \% \mathrm{CO}_{2}$ for $24 \mathrm{~h}$ and were then further cultured with $10 \mu \mathrm{L}$ of $5 \mu \mathrm{g}$ $\mathrm{mL}^{-1}$ MTT for $4 \mathrm{~h}$. Dimethyl sulfoxide (DMSO; Sigma) was added to solubilize the formazan crystals after the supernatant was discarded. After another 4-h incubation, the cell density was analyzed using an enzyme-linked immunosorbent assay reader (spectrophotometer) at $570 \mathrm{~nm}$. Each group consisted of three samples, and the reading of each was performed in triplicate to produce a mean for viability analysis.

\subsection{Caspase-3 activity assay}

Caspase-3 activity was investigated in MSCs lysates $(200 \mu \mathrm{g})$ using a Caspase-3/CPP32 Fluorometric Assay kit (BioVision, USA) according to the manufacturer's instructions. Briefly, the fluorogenic CPP32/caspase-3 substrate was labeled with the fluorochrome 7-amino-4-methyl coumarin, and then the amount of fluorescence produced upon cleavage was proportional to the amount of caspase- 3 activity present in the sample.

\section{5 miR-210 transfection}

AgomiR-210 (Ribobio, Guangzhou, China), labeled with the fluorophore 6'-FAM, was diluted to a final concentration as prescribed. According to the manufacturer's instruc- 
tions, MSCs were seeded into 24-well plates and were then transfected with agomiR-210 or the agomiR-scramble control at the indicated concentration (Ribobio), using Lipofectamine 2000 as the vehicle (Invitrogen, USA). The efficiency of transfection was determined by both quantitative-PCR and fluorescent detection.

\subsection{Quantitative polymerase chain reaction (PCR)}

microRNA was extracted from cultured cells using a mirVanaTM miRNA Isolation Kit (Ambion, USA), and complementary DNA was reverse-transcribed using a TaqMan microRNA Reverse Transcription Kit (ABI, 4366596) according to the manufacturer's protocols. The housekeeping gene miR-16 was served as internal control and the specific probes of miR-210 and miR-16 were gained from TaqMan microRNA assay kits (ABI, 4373089; ABI, 4373121). Quantitative PCR analysis was performed using TaqMan Universal PCR Master Mix (ABI, 4324018) and a 7300 real-time PCR system (Applied Biosystems, USA) according to a PCR protocol $\left(95^{\circ} \mathrm{C}\right.$ for $10 \mathrm{~min}$, then 40 cycles of $95^{\circ} \mathrm{C}$ for $15 \mathrm{~s}$, and $60^{\circ} \mathrm{C}$ for $1 \mathrm{~min}$ ). All the results were subjected to melting curve analysis, and the relative gene expressions of miR-210 were analyzed using $2^{-\Delta \Delta C_{\mathrm{t}}}$ method. All reactions were independently performed in triplicate.

\subsection{Hoechst 33342 staining}

To identify the cells, apoptosis, nuclear DNA in treated cells contained in 24-well plates was viewed by staining with Hoechst 33342, a DNA-specific dye, at a final concentration of $5 \mathrm{mg} \mathrm{mL}^{-1}$. Five fields at high magnification $(\times 200)$ were randomly chosen, and cells were immediately observed with filters for blue fluorescence, in which apoptosis index was calculated as a percentage of cells with strong blue fluorescent-nuclei to total cells.

\subsection{Determination of SOD and MDA levels}

SOD activities and MDA contents in the supernatant of cultured MSCs were measured by commercially purchased kits using colorimetric assay from Nanjing Jancheng Bioengineering Institute according to the manufacturer's instructions.

\subsection{Western blot analysis}

MSCs from each group were lysed using RIPA buffer containing $1 \mathrm{mmol} \mathrm{L}^{-1}$ phenylmethanesulfonylfluoride on ice for $30 \mathrm{~min}$ according to the methods described in the previous studies. Briefly, the lysates were centrifuged at $4^{\circ} \mathrm{C}$ and $15000 \mathrm{r} \mathrm{min}^{-1}$ for $30 \mathrm{~min}$, and then the protein component was isolated to separate by $12 \%$ SDS-PAGE followed by transfer to polyvinylidene fluoride membranes (Millipore, USA). The membranes were incubated overnight at $4^{\circ} \mathrm{C}$ with anti-phospho-c-Met or anti-c-Met (Cell Signaling, USA), and then were washed for $30 \mathrm{~min}$. After the membranes were incubated with a secondary antibody conjugated to horseradish peroxidase-conjugated IgG (Jackson, USA) for $1 \mathrm{~h}$, protein expression was determined using an enhanced chemiluminescence system and quantified by densitometry (Image System; Bio-Rad, USA).

\subsection{TUNEL (terminal dexynucleotidyl transferase- mediated dUTP nick end labeling) assay}

TUNEL was performed using the In Situ Cell Death Detection Kit (Roche, Switzerland) according to the manufacturer's protocols. In brief, MSCs were fixed in $3.7 \%$ buffered formaldehyde, pretreated with $3.0 \% \mathrm{H}_{2} \mathrm{O}_{2}$ and exposed to TdT enzyme at $37^{\circ} \mathrm{C}$ for $1 \mathrm{~h}$. After the MSCs were incubated with digoxigenin-conjugated nucleotide substrate at $37^{\circ} \mathrm{C}$ for $30 \mathrm{~min}$, nuclei exhibiting DNA fragmentation were identified after staining with 3,3-diamino benzidine (DAB) for $5 \mathrm{~min}$. The MSCs were counterstained with hematoxylin, and the percentage of labeled cells was calculated as the apoptosis index in five fields at high magnification $(\times 200)$, which were chosen randomly.

\subsection{Measurement of ROS}

The generation of intracellular ROS was evaluated using an oxidation-sensitive fluorescent dihydroethidium (DHE) probe (Vigorous, China), which can cross cell membranes and is rapidly oxidized in the presence of ROS, resulting in the formation of a highly fluorescent form of oxidative ethidium.

In this assay, MSCs were incubated with $10 \mu \mathrm{mol} \mathrm{L}{ }^{-1}$ $\mathrm{DHE}$ in phenol-red-free MEM medium (Invitrogen) at $37^{\circ} \mathrm{C}$ in the dark. After 15 min exposure, the cells were rinsed, and the medium was replaced. The fluorescence level, as an indicator of ROS accumulation, was observed using fluorescence microscopy.

\subsection{Statistical analysis}

Statistical analysis was performed using SPSS version 16.0. All data are shown as the mean \pm standard error (SE). Differences between groups were analyzed using Student's $t$-test, while comparisons between more than two groups were performed using one-way analysis of variance (ANOVA) with Bonferroni's correction. $P<0.05$ were considered to be significant.

\section{Results}

\section{1 $\mathrm{H}_{2} \mathrm{O}_{2}$ induced MSC apoptosis in a concentra- tion-dependent manner}

The MTT and caspase- 3 activity assays consistently showed 
that $\mathrm{H}_{2} \mathrm{O}_{2}$, with a culture duration of $4 \mathrm{~h}$, impaired the viability of MSCs in a dose-dependent manner over the investigated concentration range (Figure 1). A significant reduction of $26.4 \% \pm 6.32 \%$ in MSC viability and a remarkable rise of $3.68 \pm 0.38$ fold in MSC apoptosis were observed in MTT and caspase- 3 activity analysis when MSCs were exposed to $\mathrm{H}_{2} \mathrm{O}_{2}$ at a concentration of $400 \mu \mathrm{mol} \mathrm{L}{ }^{-1}(P<0.05$ for both). Therefore, we chose the above level of $\mathrm{H}_{2} \mathrm{O}_{2}$ for use in the subsequent experiments based on the results.

\section{2 miR-210 over-expression protected MSCs against $\mathrm{H}_{2} \mathrm{O}_{2}$-induced apoptosis}

Quantitative PCR was used to determine the efficiency of agomiR-210 transfection, and miR-210 was shown to be up-regulated when the concentration of agomiR-210 was over $50 \mathrm{nmol} \mathrm{L}^{-1}$ (Figure 2A). Meanwhile, there were no significant differences in cell viability when the concentration of agomiR-210 was under $200 \mathrm{nmol} \mathrm{L}^{-1}$ (Figure 2B and
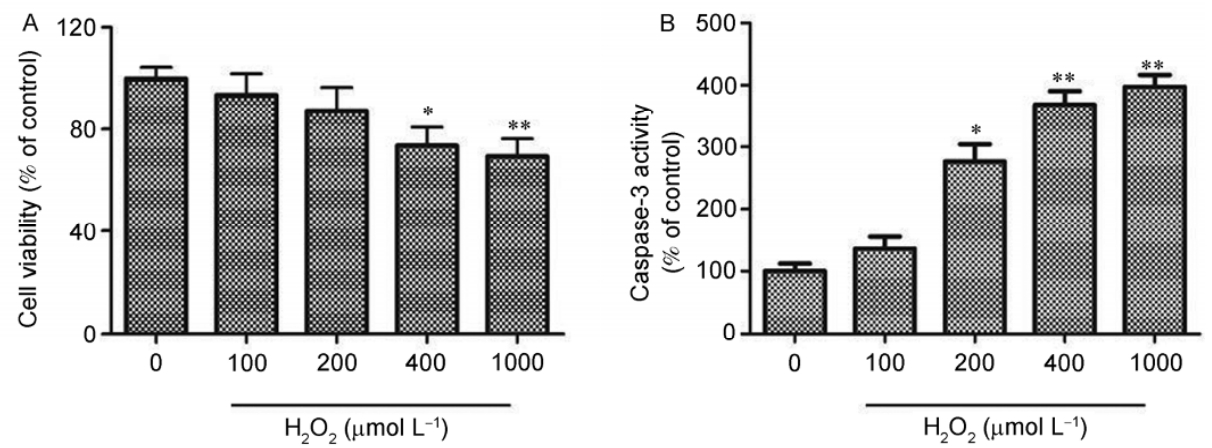

Figure 1 Effects of $\mathrm{H}_{2} \mathrm{O}_{2}$ on MSC viability and apoptosis. MSCs were cultured with increasing concentrations of $\mathrm{H}_{2} \mathrm{O}_{2}\left(0-200 \mu\right.$ mol $\left.\mathrm{L}^{-1}\right)$ for 4 h. Cell viability and apoptosis were analyzed by MTT and caspase- 3 activity assays, respectively, suggesting that $\mathrm{H}_{2} \mathrm{O}_{2}$ decreased MSC viability (A) and induced MSCs apoptosis (B) in a concentration-dependent manner. The results are from three independent experiments. *, $P<0.05 \mathrm{vs.}$ control; $* *, P<0.01$ vs. control.
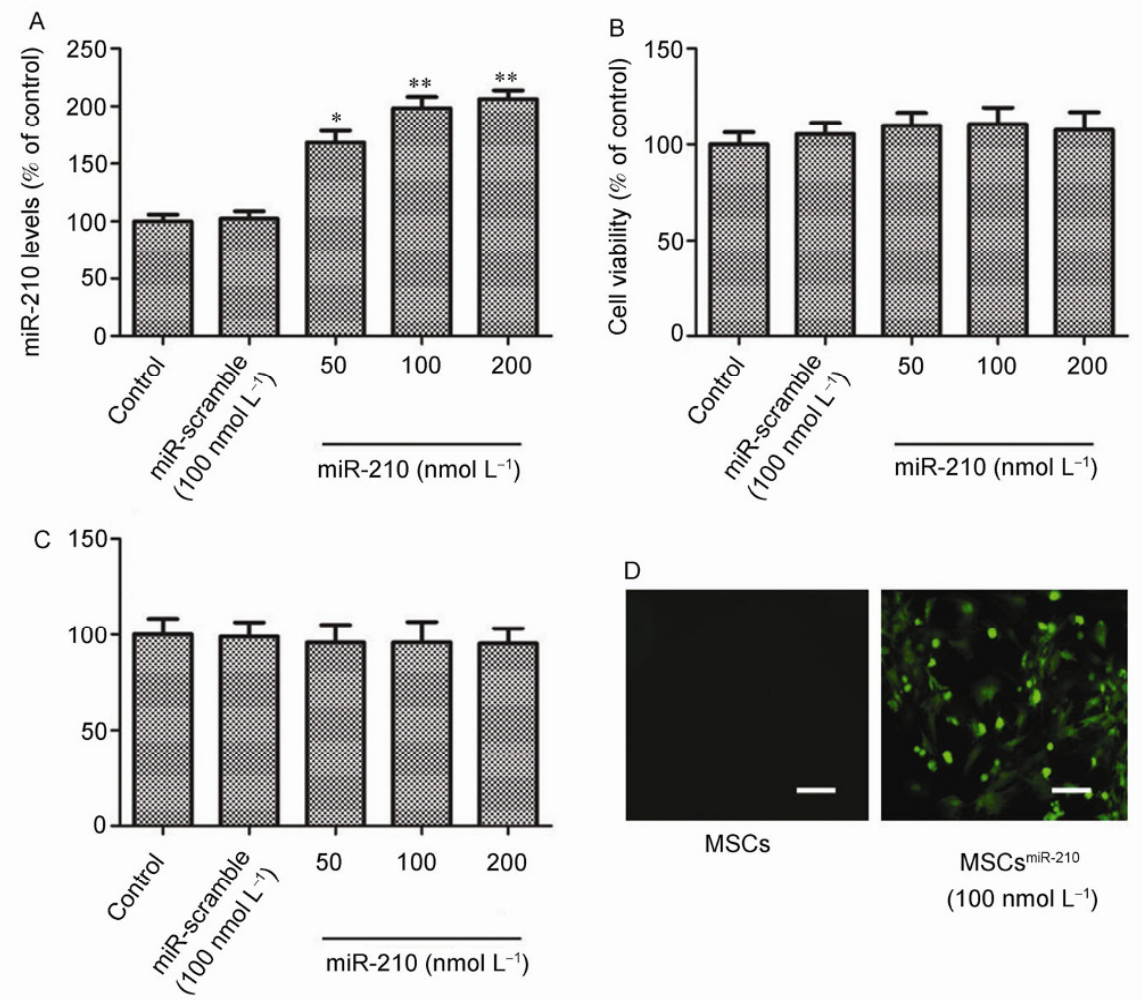

Figure 2 Effects of miR-210 over-expression on MSC viability. MSCs were transfected with increasing doses of agomiR-210 $\left(0-200 \mathrm{nmol} \mathrm{L}^{-1}\right)$ or with agomiR-scramble $\left(100 \mathrm{nmol} \mathrm{L}^{-1}\right)$, the negative control. Quantitative-PCR was performed to verify the efficacy of miR-210 transfection (A); meanwhile, MTT and caspase-3 activity were measured to investigate the effects of miR-210 over-expression on MSC viability (B and C). AgomiR-210 in a concentration of $100 \mathrm{nmol} \mathrm{L}^{-1}$ was selected for the subsequent transfection experiments, and a representative image, visualized by fluorescent microscopy, is shown (D; scale bar, $20 \mu \mathrm{m})$. Data were from three independent experiments. *, $P<0.05$ vs. control; **, $P<0.01$ vs. control. 
C) (all $P>0.05$ ). Based on the above data, agomiR-210 at a concentration of $100 \mathrm{nmol} \mathrm{L}^{-1}$ was chosen for use in the subsequent experiments, and a representative image, visualized by fluorescent microscopy, is shown (Figure 2D).

To investigate the effects of miR-210 over-expression on $\mathrm{H}_{2} \mathrm{O}_{2}$-induced MSC apoptosis, MSCs, over-expressing miR-210 or not, were cultured with $400 \mu$ mol L ${ }^{-1} \mathrm{H}_{2} \mathrm{O}_{2}$ for $4 \mathrm{~h}$. In comparisons of the MSCs ${ }^{\text {miR-210 }}+\mathrm{H}_{2} \mathrm{O}_{2}$ group with the MSCs $+\mathrm{H}_{2} \mathrm{O}_{2}$ group, apoptosis in $\mathrm{H}_{2} \mathrm{O}_{2}$-insulted MSCs was dramatically reduced by miR-210 over-expression, as shown by Hoechst 33342 staining $(153.2 \% \pm 17.3 \%$ vs. $276.4 \% \pm 19.4 \%, P<0.05)$, the MTT assay $(86.9 \% \pm 8.33 \%$ vs. $72.2 \% \pm 6.14 \%, P<0.05)$, and caspase-3 activity $(153.7 \% \pm$ $11.4 \%$ vs. $352.5 \% \pm 13.8 \%, P<0.05$ ) (Figure 3 ).

\section{3 miR-210 over-expression enhances MSC survival through antioxidation}

The examination of SOD activities in the supernatant of MSC cultures demonstrated that there was a significant reduction in the MSCs $+\mathrm{H}_{2} \mathrm{O}_{2}$ group compared with the MSCs group $(47.3 \% \pm 9.27 \%$ vs. $100.0 \% \pm 7.32 \%, P<0.05)$, and this reduction was remarkably reversed in the $\mathrm{MSCs}^{\mathrm{miR}-210}+$ $\mathrm{H}_{2} \mathrm{O}_{2}$ group $(P<0.05)$ (Figure 4A). Consistently, there was a dramatic increase in the MDA level in the media in the
MSCs $+\mathrm{H}_{2} \mathrm{O}_{2}$ group compared to the MSCs group (286.3\% \pm $7.53 \%$ vs. $100.0 \% \pm 9.31 \%, P<0.05)$. However, the MDA levels were significantly decreased in the MSCs ${ }^{\text {miR-210 }}+\mathrm{H}_{2} \mathrm{O}_{2}$ group compared to the $\mathrm{MSCs}+\mathrm{H}_{2} \mathrm{O}_{2}$ group $(167.4 \% \pm 9.25 \%$ vs. $286.3 \% \pm 7.53 \%, P<0.05$ ) (Figure 4B). Meanwhile, there were no significant differences in either the SOD activities or the MDA levels of the MSCs and MSCs ${ }^{\text {miR-210 }}$ groups (both $P>0.05$ ).

\section{4 miR-210 over-expression attenuates the c-Met ac- tivity repression induced by $\mathrm{H}_{2} \mathrm{O}_{2}$}

Western blot analysis was used to assess the changes in activity of the c-Met pathway. The results indicated that transfection of the agomiR-scramble did not significantly affect the phosphorylation of c-Met $(P>0.05)$, while over-expression of miR-210 significantly up-regulated the activity of the c-Met pathway $(P<0.05)$. Oxidative stress dramatically suppressed the activation of the c-Met pathway (MSCs $+\mathrm{H}_{2} \mathrm{O}_{2}$ group vs. MSCs group: $32.4 \% \pm 6.73 \%$ vs. $100.0 \% \pm 13.8 \%, P<0.05$ ) ; however, miR-210 over-expression significantly restored the down-regulation of c-Met phosphorylation in MSCs stimulated by $\mathrm{H}_{2} \mathrm{O}_{2}\left(\mathrm{MSCs}^{\text {miR-210 }}\right.$ $+\mathrm{H}_{2} \mathrm{O}_{2}$ group vs. $\mathrm{MSCs}+\mathrm{H}_{2} \mathrm{O}_{2}$ group: $72.8 \% \pm 10.3 \%$ vs. $32.4 \% \pm 6.73 \%, P<0.05$ ) (Figure 5A).
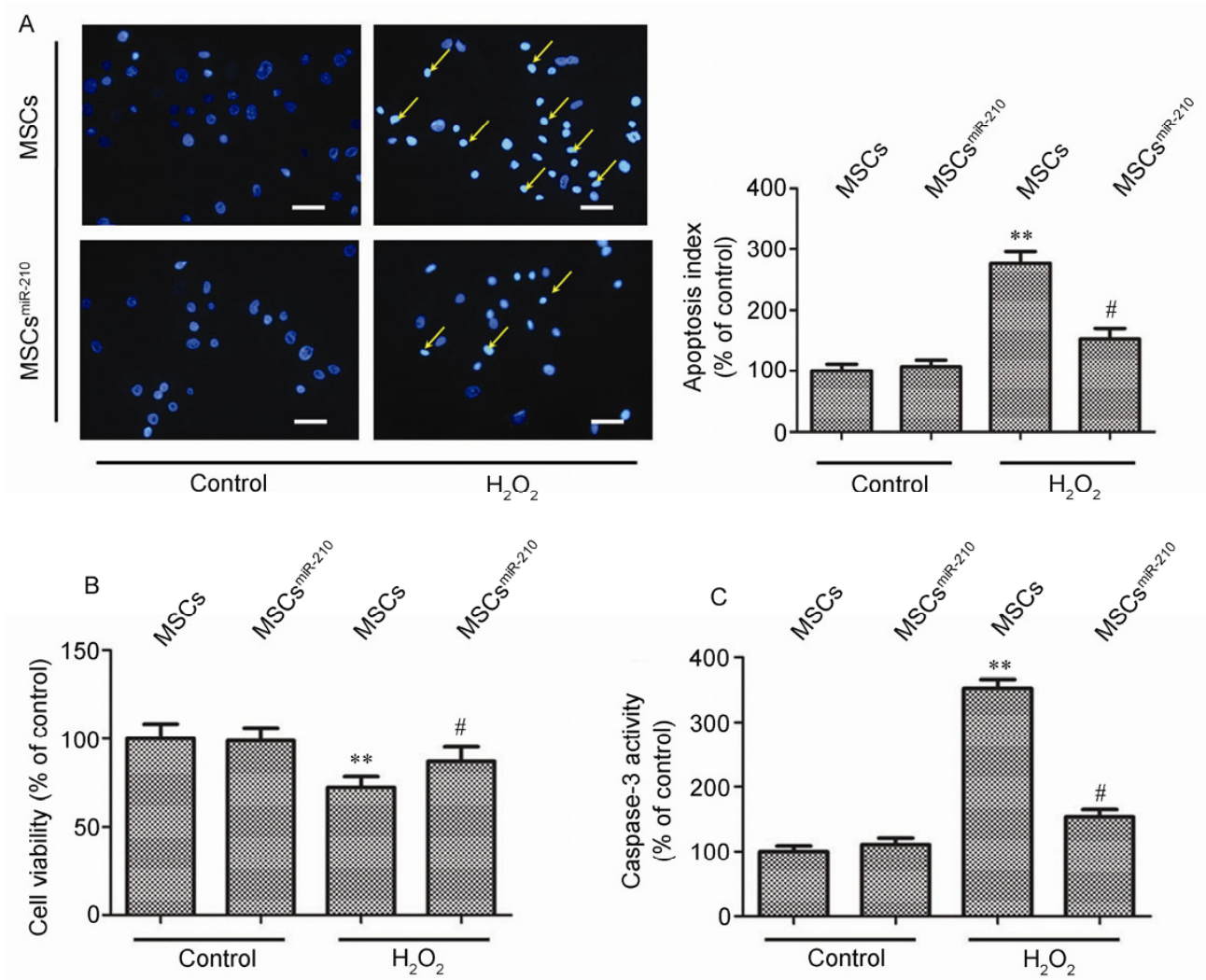

Figure 3 Effects of miR-210 on $\mathrm{H}_{2} \mathrm{O}_{2}$-induced MSC apoptosis. MSCs ${ }^{\text {miR-210 }}$ or MSCs were incubated with or without $\mathrm{H}_{2} \mathrm{O}_{2}\left(100 \mu\right.$ mol $\left.\mathrm{L}^{-1}\right)$ for 4 h. Cells apoptosis was measured with Hoechst 33342, MTT and caspase-3 activity assays as shown in photomicrograph A (scale bar, $20 \mu \mathrm{m}$ ) and in histograms B and C. The results are from three independent experiments. *, $P<0.05$ vs. MSCs group; **, $P<0.01$ vs. MSCs group; \#, $P<0.05$ vs. $\mathrm{MSCs}+\mathrm{H}_{2} \mathrm{O}_{2}$ group. 

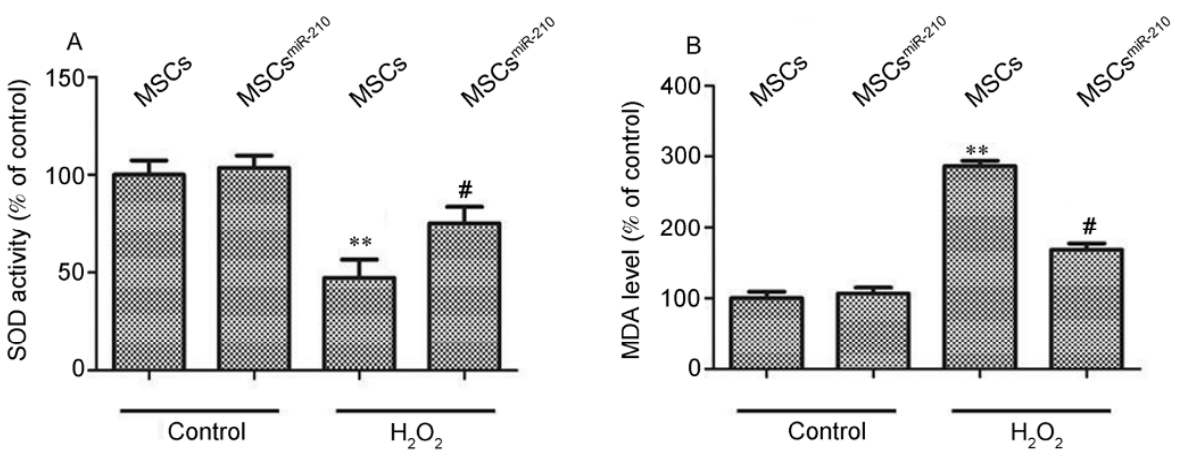

Figure 4 Antioxidation contributes to the protective effect of miR-210 in MSCs. MSCs were incubated with $\mathrm{H}_{2} \mathrm{O}_{2}\left(100 \mu \mathrm{mol} \mathrm{L} \mathrm{L}^{-1}\right)$ for $4 \mathrm{~h}$ following transfection with miR-210, and miR-210 over-expression abolished the decrease of SOD activity and the increase of MDA induced by $\mathrm{H}_{2} \mathrm{O}_{2}$. Data were from three independent experiments, presented as the mean $\pm \mathrm{SD}$ and shown in histograms (A and $\mathrm{B}$ ). *, $P<0.05$ vs. MSCs group; **, $P<0.01$ vs. MSCs group; \#, $P<0.05$ vs. MSCs treated with $\mathrm{H}_{2} \mathrm{O}_{2}$ group.
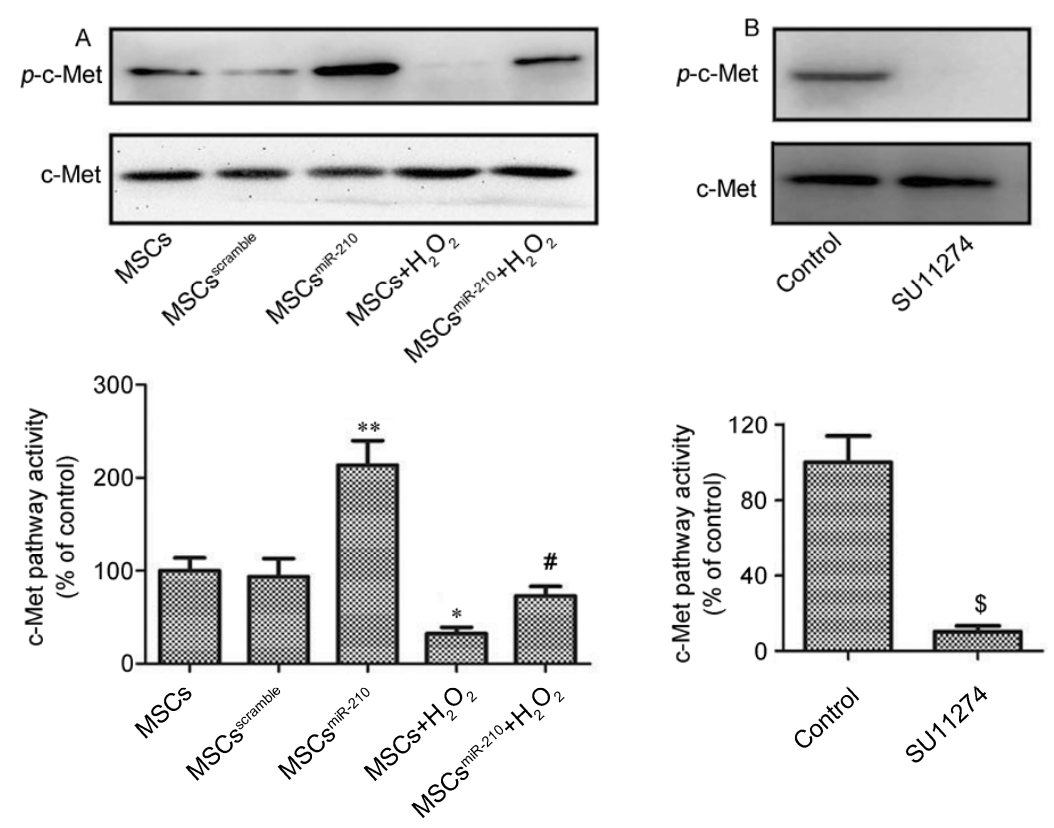

Figure 5 The c-Met pathway is involved in the anti-apoptotic protection of miR-210 in MSCs. Western blotting was used to assess c-Met phosphorylation, indicating c-Met pathway activation, and the results were quantified and exhibited as histograms. A, MSCs were exposed to $\mathrm{H}_{2} \mathrm{O}_{2}\left(100 \mu \mathrm{mol} \mathrm{L} \mathrm{L}^{-1}\right)$ for $4 \mathrm{~h}$ after transfection with miR-210, with miR-scramble, or with no miRNAs. B, SU11274 was used to inhibit c-Met pathway activity, and the efficacy of inhibition was investigated. *, $P<0.05$ vs. MSCs group; **, $P<0.01$ vs. MSCs group; $\#, P<0.05$ vs. MSCs treated with $\mathrm{H}_{2} \mathrm{O}_{2}$ group; $\$, P<0.05$ vs. control group.

\subsection{The c-Met pathway mediates the protective effect of miR-210 on MSC apoptosis induced by $\mathrm{H}_{2} \mathrm{O}_{2}$}

Western blot analysis suggested that SU11274 $\left(10 \mu \mathrm{mol} \mathrm{L}^{-1}\right)$ preconditioning obviously suppressed c-Met activation in MSCs (Figure 5B), which reversed the protective effects of miR-210 over-expression on $\mathrm{H}_{2} \mathrm{O}_{2}$-induced cells' apoptosis. The TUNEL assay indicated that the apoptosis rate was significantly up-regulated in the $\mathrm{MSCs}^{\mathrm{miR}-210}+\mathrm{H}_{2} \mathrm{O}_{2}+$ SU11274 group compared to that in the MSCs ${ }^{\text {miR-210 }}+\mathrm{H}_{2} \mathrm{O}_{2}$ group $(216.7 \% \pm 17.3 \%$ vs. $156.8 \% \pm 11.5 \%, P<0.05)$, while there was no significant difference between the MSCs $+\mathrm{SU} 11274$ and MSCs groups $(P>0.05)$ and between the $\mathrm{MSCs}^{\text {miR-210 }}+\mathrm{H}_{2} \mathrm{O}_{2}$ and MSCs ${ }^{\text {miR-210 }}+\mathrm{H}_{2} \mathrm{O}_{2}+$ DMSO groups
$(P>0.05)$ (Figure 6).

Furthermore, SU11274 pretreatment was shown to abolish the beneficial effect of miR-210 over-expression on $\mathrm{H}_{2} \mathrm{O}_{2}$-induced ROS accumulation in MSCs. The number of ROS negative cells, with low fluorescence intensity, were similar between the MSCs+SU11274 and MSCs groups $(P>0.05)$; additionally, the number of cells with strong red fluorescence, indicating ROS accumulation, were not significantly different between the $\mathrm{MSCS}^{\text {miR-210 }}+\mathrm{H}_{2} \mathrm{O}_{2}$ and $\mathrm{MSCs}^{\mathrm{miR}-210}+\mathrm{H}_{2} \mathrm{O}_{2}+\mathrm{DMSO}$ groups $(P>0.05)$. However, intracellular ROS generation in the MSCs ${ }^{\text {miR-210 }}+\mathrm{H}_{2} \mathrm{O}_{2}+$ SU11274 group was remarkably higher than that in the MSCs ${ }^{\text {miR-210 }}+\mathrm{H}_{2} \mathrm{O}_{2}$ group $(492.7 \% \pm 26.4 \%$ vs. $176.4 \% \pm$ $19.7 \%, P<0.05$ ) (Figure 7). 


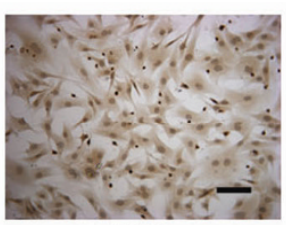

MSCs

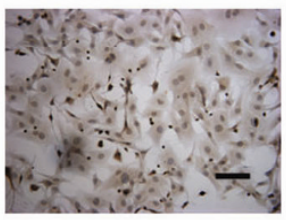

MSCs $^{\text {miR-210+DMSO }}$

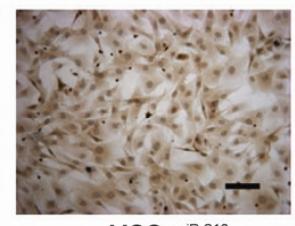

MSCs $s^{\text {miR-210 }}$

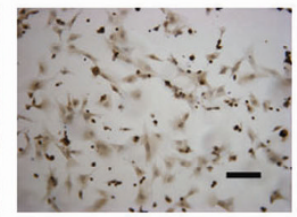

$\mathrm{MSCs}^{\mathrm{miR}-210}+\mathrm{SU} 11274$

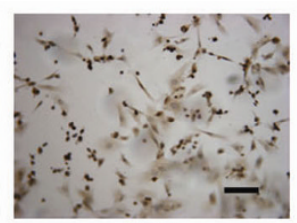

MSCs
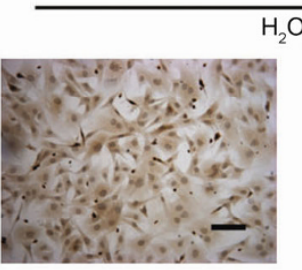

MSCs+SU11274

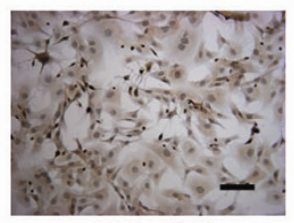

MSCs $^{\text {miR-210 }}$

$\mathrm{H}_{2} \mathrm{O}_{2}$

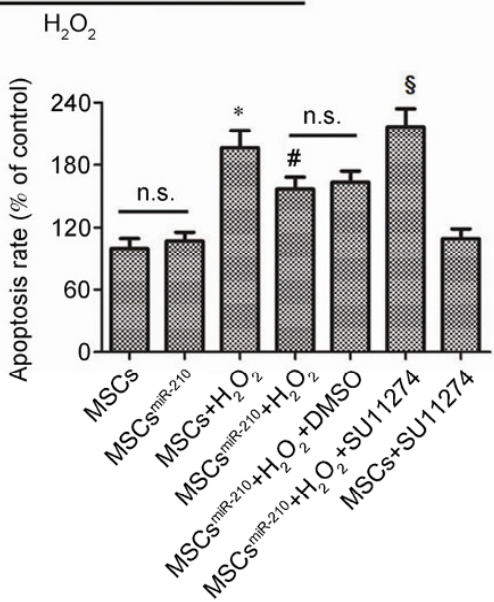

Figure 6 Role of the c-Met pathway in the protective effect of miR-210 in MSCs against apoptosis induced by $\mathrm{H}_{2} \mathrm{O}_{2}$. TUNEL was performed to assess apoptosis, in which cells with brown staining, the TUNEL-positive cells, were counted, and the apoptotic rate was calculated as the percentage of apoptotic cells. Representative photomicrographs (scale bar, $50 \mu \mathrm{m}$ ) and corresponding histograms are shown. Data were from three independent experiments. *, $P<0.05$ vs. MSCs group; \#, $P<0.05$ vs. MSCs $+\mathrm{H}_{2} \mathrm{O}_{2}$ group; $\S, P<0.05$ vs. MSCs ${ }^{\text {miR-210 }}+\mathrm{H}_{2} \mathrm{O}_{2}$ group; n.s., not significant.

\section{Discussion}

Transplantation of mesenchymal stem cells has been reported to be an attractive approach for cardiovascular therapy because of the capacity of these cells to facilitate myocardial repair in models of cardiac injury, such as myocardial infarction [1]. However, this approach has been shown to be limited because even when $6 \times 10^{7}$ MSCs were implanted in infarcted porcine hearts, only a slight improvement of LVEF occurred [2]. The poor viability of MSCs exposed to oxidative stress, hypoxia and inflammatory attack greatly restricted the efficacy of cell-based treatment [17], which prompted the exploration of a series of strategies to prevent peri-transplantation graft cell death.

microRNAs (miRNAs) regulate gene expression at a post-transcriptional level by inhibiting mRNA translation or promoting mRNA degradation [19] and are increasingly reported to play major roles in the death or apoptosis of cells $[11,12]$. Multiple studies have shown that miR-210 is closely involved with hypoxic response and oxidative stress regulation in cancer cells, and high levels of miR-210 have been linked to an adverse prognosis in cancer patients [20,21]. A wide spectrum of miR-210 targets have been shown to be involved in mitochondrial metabolism, oxidative stress and cell survival, which broadly affect a variety of pathological settings, such as cancer and ischemic disorders [22]. However, whether miR-210 can influence MSC survival in oxidative stress conditions and the underlying molecular mechanism are not yet fully understood.

Therefore, we attempted to clarify whether overexpression of miR-210 in MSCs could enhance cell viability and reduce oxidative stress-induced apoptosis. In the present study, we showed that miR-210 over-expression in MSCs suppressed the MDA level and increased SDA activity, thus protecting MSCs from $\mathrm{H}_{2} \mathrm{O}_{2}$-induced apoptosis in vitro. The mechanism involved the activation of c-Met, an upstream factor of the PI3K/Akt pathway, which was regarded as pivotal in controlling cell survival. We also demonstrated that inhibition of the c-Met pathway reversed the beneficial effect of miR-210, indicating that c-Met activation was the key mechanism for the protective role of miR-210 in MSC survival under oxidative stress, which 


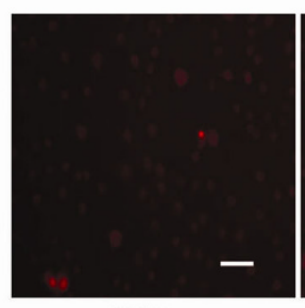

MSCs
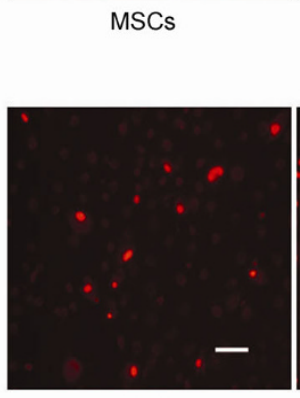

MSCs $^{\text {miR-210+DMSO }}$

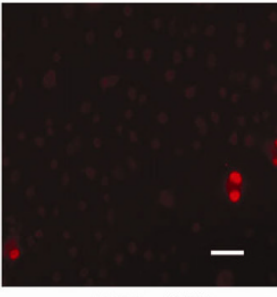

MSCs $^{\text {miR-210 }}$

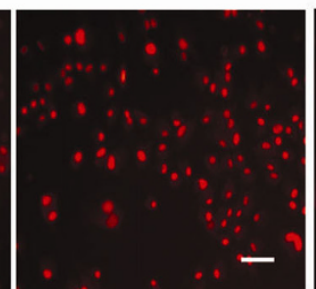

MSCs

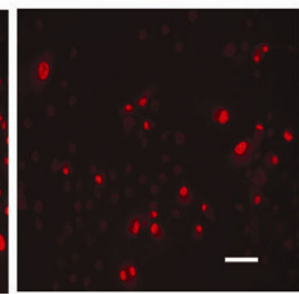

$\mathrm{MSCs}^{\mathrm{miR}-210}$

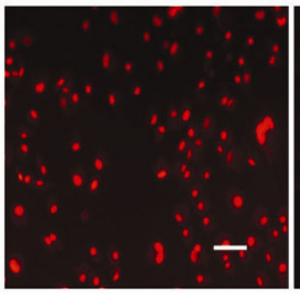

$\mathrm{MSCs}^{\mathrm{miR}-210+S U 11274}$

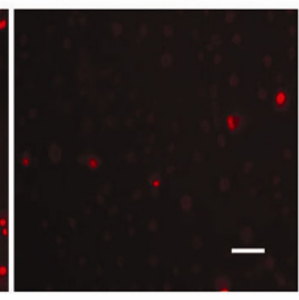

MSCs+SU11274

$\mathrm{H}_{2} \mathrm{O}_{2}$

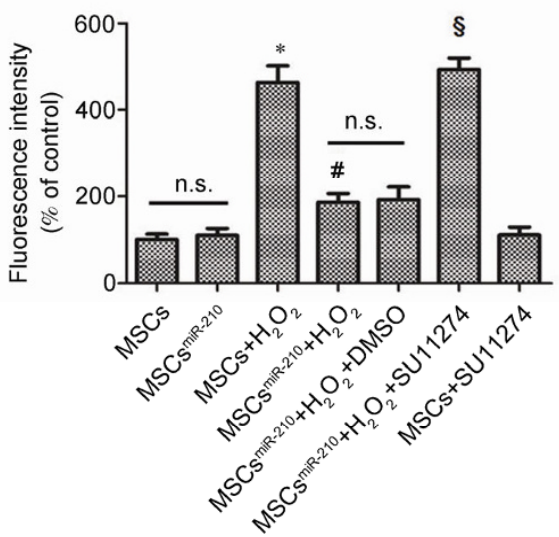

Figure 7 Impact of the c-Met pathway on the protective effect of miR-210 against ROS accumulation induced by $\mathrm{H}_{2} \mathrm{O}_{2}$. The intracellular ROS in MSCs was visualized by the fluorescent probe DHE. Hence, cells with strong red fluorescence indicated ROS accumulation while those with low fluorescence intensity were regarded as ROS-negative. The entire fluorescence intensity of each photomicrography was quantified and compared. Representative photomicrographs (scale bar, $50 \mu \mathrm{m}$ ) and corresponding histograms are displayed. The results are from three independent experiments. $*, P<0.05$ vs. MSCs group; $\#, P<0.05$ vs. MSCs $+\mathrm{H}_{2} \mathrm{O}_{2}$ group; $\S, P<0.05$ vs. MSCs ${ }^{\text {miR-210 }}+\mathrm{H}_{2} \mathrm{O}_{2}$ group; ns, not significant.

down-regulated the phosphorylation of c-Met. Additionally, we showed that suppressing the c-Met pathway abolished the restraining function of miR-210 on ROS production induced by $\mathrm{H}_{2} \mathrm{O}_{2}$, suggesting that c-Met phosphorylation occurs upstream of ROS generation. Based on previous studies showing that excessive accumulation of ROS has been shown to damage stem cells through JNK- and caspase-mediated mechanisms [23], it could be suggested that over-expression of miR-210 in MSCs decreased intracellular oxidative reactions through the activation of the c-Met pathway, thus leading to an obvious improvement of cell viability under oxidative stress.

In stem cells, miR-210 might play a critical function that links apoptosis signals to the oxidative stress microenvironment, which is the vital finding in our study and was consistently shown by previous reports [24,25]. Favaro et al. [21] showed that ROS accumulation could be alleviated with antimiR-210 treatment in cancer cell lines. Furthermore, Chan et al. [20] demonstrated that, in normal endothelial cells, they could not detect any significant change in ROS generation after exposure to hypoxia, which increased when miR-210 was down-regulated. Elucidating the role of miR-210 in MSC apoptosis induced by oxidative stress may lead to novel therapeutic approaches that enhance the efficacy of MSC-based treatment for injured myocardium, which was the most clinically significant conclusion from our results.

The novelty of the present study is that we explored a probable modification of MSCs that improves cell viability under oxidative stress. Identifying the diverse signatures and functions of miRNAs in MSC pathophysiology may help fully elucidate the precise modulatory mechanism when pro-apoptotic factors are present; a clear understanding of the role of miR-210 in this process may be a step in 
this direction.

In the current study, we did not further investigate, in vivo, whether the survival of MSCs over-expressing miR-210 could be enhanced in the infarcted myocardium. This will be the subject of future research, along with an observation of other underlying effects of miR-210 on MSCs (such as cell proliferation, migration and secretion), which may be critical in restoring cardiac function.

In summary, we demonstrated that miR-210 overexpression protected against $\mathrm{H}_{2} \mathrm{O}_{2}$-induced apoptosis in MSCs by antioxidation and the up-regulation of the c-Met pathway. These discoveries provide an experimental basis for the development of MSC-based treatment for ischemic heart disease and for thorough clarification of MSC survival regulation from a miRNA-modulation perspective.

This work was supported by the National Natural Science Foundation of China (81100145, 81370003, 81300082, 81370322), the China Postdoctoral Science Foundation funded project (2013M531124, 2014T70391), the Cardiovascular Research Fund supported by Chinese Association of Physicians (DFCMDA201259, DFCMDA201255), and the Key Specialty Construction of Medical Program in Shanghai (ZK2012A24).

1 Dong F, Harvey J, Finan A, Weber K, Agarwal U, Penn MS. Myocardial CXCR4 expression is required for mesenchymal stem cell mediated repair following acute myocardial infarction. Circulation, 2012, 126: 314-324

2 Wang XH, Zhao TM, Huang W, Wang T, Qian J, Xu MF, Kranias EG, Wang YG, Fan GC. Hsp20-engineered mesenchymal stem cells are resistant to oxidative stress via enhanced activation of Akt and increased secretion of growth factors. Stem Cells, 2009, 27: 3021-3031

3 Wang LH, Hu XY, Zhu W, Jiang Z, Zhou Y, Chen PP, Wang JA. Increased leptin by hypoxic-preconditioning promotes autophagy of mesenchymal stem cells and protects them from apoptosis. Sci China Life Sci, 2014, 57: 171-180

4 Liu XH, Bai CG, Xu ZY, Huang SD, Yuan Y, Gong DJ, Zhang JR. Therapeutic potential of angiogenin modified mesenchymal stem cells: Angiogenin improves mesenchymal stem cells survival under hypoxia and enhances vasculogenesis in myocardial infarction. Microvasc Res, 2008, 76: 23-30

5 Cheng Y, Qiu F, Ye YC, Guo ZM, Tashiro SI, Onodera S, Ikejima T. Autophagy inhibits reactive oxygen species-mediated apoptosis via activating p38-nuclear factor-kappa $B$ survival pathways in oridonin-treated murine fibrosarcoma L929 cells. FEBS J, 2009, 276: 1291-1306

6 Panieri E, Gogvadze V, Norberg E, Venkatesh R, Orrenius S, Zhivotovsky B. Reactive oxygen species generated in different compartments induce cell death, survival, or senescence. Free Rad Biol Med, 2013, 57: 176-187

7 Li JR, Wu Y, Qi YJ. microRNAs in a multicellular green alga Volvox carteri. Sci China Life Sci, 2014, 57: 36-45

8 Wu D, Hu Y, Tong S, Williams BRG, Smyth GK, Gantier MP. The use of miRNA microarrays for the analysis of cancer samples with global miRNA decrease. RNA (New York, NY), 2013, 19: 876-888

9 Balasubramanyam M, Aravind S, Gokulakrishnan K, Prabu P, Sathishkumar C, Ranjani H, Mohan V. Impaired miR-146a expression links subclinical inflammation and insulin resistance in Type 2 diabetes. Mol Cell Biochem, 2011, 351: 197-205
10 Huang F, Zhu X, Hu XQ, Fang ZF, Tang L, Lu XL, Zhou SH. Mesenchymal stem cells modified with miR-126 release angiogenic factors and activate Notch ligand Delta-like-4, enhancing ischemic angiogenesis and cell survival. Int J Mol Med, 2013, 31: 484-492

11 Luft FC. Merely miR210 in mesenchymal stem cells-one size fits all. J Mol Med (Berl), 2012, 90: 983-985

12 Cicchillitti L, Di Stefano V, Isaia E, Crimaldi L, Fasanaro P, Ambrosino V, Antonini A, Capogrossi MC, Gaetano C, Piaggio G. Hypoxia-inducible factor 1-alpha induces miR-210 in normoxic differentiating myoblasts. J Biol Chem, 2012, 287: 44761-44771

13 Zhuang HQ, Bo QF, Yuan ZY, Wang J, Zhao LJ, Wang P. The different radiosensitivity when combining erlotinib with radiation at different administration schedules might be related to activity variations in c-MET-PI3K-AKT signal transduction. Oncotarg Ther, 2013, 6: 603-608

14 Lai L, Yan L, Gao SM, Hu CL, Ge H, Davidow A, Park M, Bravo C, Iwatsubo K, Ishikawa Y. Type 5 adenylyl cyclase increases oxidative stress by transcriptional regulation of manganese superoxide dismutase via the SIRT1/FoxO3a pathway. Circulation, 2013, 127: 1692-1701

15 Wen YD, Wang H, Kho SH, Rinkiko S, Sheng X, Shen HM, Zhu YZ. Hydrogen sulfide protects HUVECs against hydrogen peroxide induced mitochondrial dysfunction and oxidative stress. PLoS One, 2013, 8: e53147

16 Xu J, Qian J, Xie X, Lin L, Zou Y, Fu M, Huang Z, Zhang G, Su Y, Ge J. High density lipoprotein protects mesenchymal stem cells from oxidative stress-induced apoptosis via activation of the PI3K/Akt pathway and suppression of reactive oxygen species. Int J Mol Sci, 2012, 13: 17104-17120

17 Xu J, Qian J, Xie X, Lin L, Ma J, Huang Z, Fu M, Zou Y, Ge J. High density lipoprotein cholesterol promotes the proliferation of bone-derived mesenchymal stem cells via binding scavenger receptor-B type I and activation of PI3K/Akt, MAPK/ERK1/2 pathways. Mol Cell Biochem, 2012, 371: 55-64

18 Jung HY, Joo HJ, Park JK, Kim YH. The Blocking of c-Met signaling induces apoptosis through the increase of $\mathrm{p} 53$ protein in lung cancer. Cancer Res Treat, 2012, 44: 251-261

19 Zhao N, Yu H, Yu H, Sun M, Zhang Y, Xu M, Gao W. miRNA-711-SP1-collagen-I pathway is involved in the anti-fibrotic effect of pioglitazone in myocardial infarction. Sci China Life Sci, 2013, 56: 431-439

20 Chan SY, Loscalzo J. Nitric oxide up-regulates microRNA-210 to repress iron-sulfur cluster-dependent mitochondrial metabolism in vascular endothelial cells. Circulation, 2010, 122: A11386

21 Favaro E, Ramachandran A, McCormick R, Gee H, Blancher C, Crosby M, Devlin C, Blick C, Buffa F, Li JL. microRNA-210 regulates mitochondrial free radical response to hypoxia and krebs cycle in cancer cells by targeting iron sulfur cluster protein ISCU. PLoS One, 2010, 5: e10345

22 Chan YC, Banerjee J, Choi SY, Sen CK. miR-210: the master hypoxamir. Microcirculation, 2012, 19: 215-223

23 Tam Dan Nguyen N, Son YO, Lim SS, Shi X, Kim JG, Heo JS, Choe Y, Jeon YM, Lee JC. Sodium fluoride induces apoptosis in mouse embryonic stem cells through ROS-dependent and caspase- and JNK-mediated pathways. Toxicol Appl Pharmacol, 2012, 259: 329-337

24 Wang F, Xiong L, Huang X, Zhao T, Wu LY, Liu ZH, Ding X, Liu S, Wu Y, Zhao Y. miR-210 suppresses BNIP3 to protect against the apoptosis of neural progenitor cells. Stem Cell Res, 2013, 11: 657-667

25 McCormick RI, Blick C, Ragoussis J, Schoedel J, Mole DR, Young AC, Selby PJ, Banks RE, Harris AL. miR-210 is a target of hypoxia-inducible factors 1 and 2 in renal cancer, regulates ISCU and correlates with good prognosis. British J Cancer, 2013, 108: 1133-1142

Open Access This article is distributed under the terms of the Creative Commons Attribution License which permits any use, distribution, and reproduction in any medium, provided the original author(s) and source are credited. 